\title{
In vivo performance of a microelectrode neural probe with integrated drug delivery
}

\author{
Laboratory investigation
}

\author{
Pratik Rohatgi, M.S.E., ${ }^{1,2}$ Nicholas B. Langhals, M.S.E., ${ }^{2}$ Daryl R. Kipke, Ph.D., ${ }^{2}$ \\ and Parag G. Patil, M.D., Ph.D. ${ }^{1-3}$ \\ ${ }^{1}$ University of Michigan Medical School, and Departments of ${ }^{2}$ Biomedical Engineering and ${ }^{3}$ Neurosurgery, \\ University of Michigan, Ann Arbor, Michigan
}

\begin{abstract}
Object. The availability of sophisticated neural probes is a key prerequisite in the development of future brainmachine interfaces (BMIs). In this study, the authors developed and validated a neural probe design capable of simultaneous drug delivery and electrophysiology recordings in vivo. Focal drug delivery promises to extend dramatically the recording lives of neural probes, a limiting factor to clinical adoption of BMI technology.

Methods. To form the multifunctional neural probe, the authors affixed a 16-channel microfabricated silicon electrode array to a fused silica catheter. Three experiments were conducted in rats to characterize the performance of the device. Experiment 1 examined cellular damage from probe insertion and the drug distribution in tissue. Experiment 2 measured the effects of saline infusions delivered through the probe on concurrent electrophysiological measurements. Experiment 3 demonstrated that a physiologically relevant amount of drug can be delivered in a controlled fashion. For these experiments, Hoechst and propidium iodide stains were used to assess insertion trauma and the tissue distribution of the infusate. Artificial CSF (aCSF) and tetrodotoxin (TTX) were injected to determine the efficacy of drug delivery.

Results. The newly developed multifunctional neural probes were successfully inserted into rat cortex and were able to deliver fluids and drugs that resulted in the expected electrophysiological and histological responses. The damage from insertion of the device into brain tissue was substantially less than the volume of drug dispersion in tissue. Electrophysiological activity, including both individual spikes as well as local field potentials, was successfully recorded with this device during real-time drug delivery. No significant changes were seen in response to delivery of aCSF as a control experiment, whereas delivery of TTX produced the expected result of suppressing all spiking activity in the vicinity of the catheter outlet.

Conclusions. Multifunctional neural probes such as the ones developed and validated within this study have great potential to help further understand the design space and criteria for the next generation of neural probe technology. By incorporating integrated drug delivery functionality into the probes, new treatment options for neurological disorders and regenerative neural interfaces using localized and feedback-controlled delivery of drugs can be realized in the near future. (DOI: 10.3171/2009.4.FOCUS0983)
\end{abstract}

\section{KEY WORDS • drug delivery • neural engineering • rat microelectrode array}

$\mathrm{B}$ RAIN-MACHINE interfaces depend on technology that detects neural activity in the CNS. Neural firing patterns are often recorded from microelectrodes, which in turn are used to measure an output signal that can be manipulated by a patient's brain activity. ${ }^{4,10,11,41} \mathrm{~A}$ new avenue to interface with the CNS can be achieved by providing drug delivery capabilities through existing neural probe technology.

A large barrier to the clinical adoption of BMI technologies is electrode failure. Device failure is hypothe-

\footnotetext{
Abbreviations used in this paper: $\mathrm{aCSF}=$ artificial $\mathrm{CSF} ; \mathrm{BMI}=$ brain-machine interface; $\mathrm{CED}=$ convection-enhanced delivery; LFP $=$ local field potential; PI = propidium iodide; TTX = tetrodotoxin .
}

sized to result from a foreign-body response that gradually encapsulates the BMI implant with scar tissue. $17,30,33$ This process increases the distance between electrode sites and neuronal sources, reducing the ability to record brain activity. Systemic corticosteroid delivery has been investigated to mitigate this inflammatory process; however, medication side effects limit the duration and strength of the therapy.$^{38}$ Systemic drug delivery requires large administered doses to penetrate the blood-brain barrier at a sufficient concentration to have a measurable effect in the brain. Consequently, there is a great interest in controlled and targeted drug delivery to the brain, so that small, localized doses can be used instead of large, systemic drug doses. Because the reactive tissue 


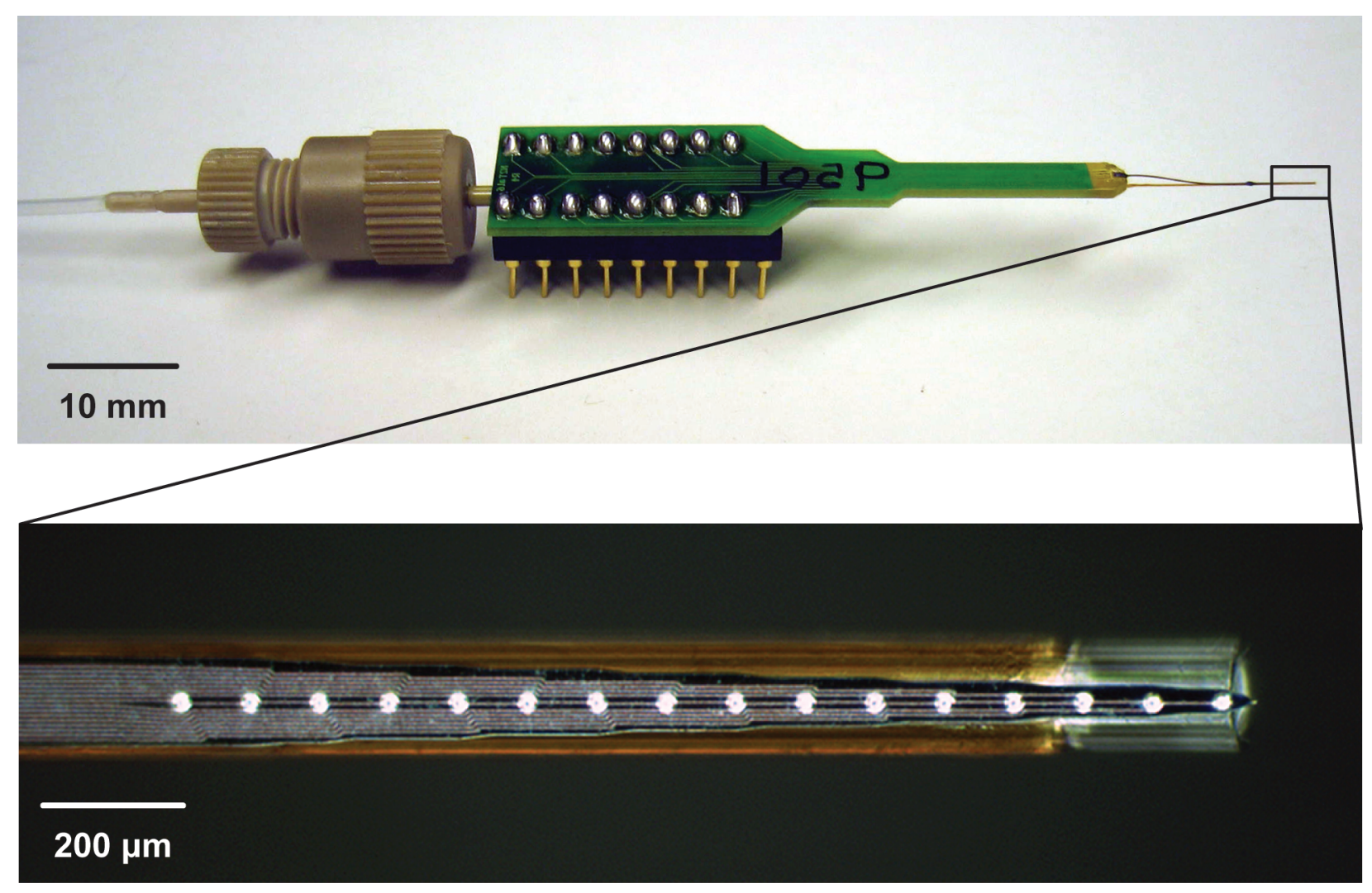

FIG. 1. Upper: Photograph showing the full neural probe and attached fluidic line. Lower: A magnified view showing the electrode sites on the array. Each of the 16 iridium microelectrode sites have a surface area of $703 \mu \mathrm{m}^{2}$ and are spaced 100 $\mu \mathrm{m}$ apart. The catheter is made of fused silica covered in a polyimide sheath, with an outer diameter of $165 \mu \mathrm{m}$ and an inner diameter of $127 \mu \mathrm{m}$.

responses against implanted neural prostheses are focal by nature, it may be fruitful to use focal interventional strategies to mitigate this process.

Because nervous system processes are driven by both chemical and electrical signals, multimodal therapies may have applications in the future. In fact, systemic drug delivery is still the principal treatment for many neurological disorders. For example, Parkinson disease treatment might be advanced by adding a drug delivery capability to deep brain stimulating electrodes, potentially reducing the need for systemic medications and the associated side effects. Epilepsy treatment devices may be envisioned to provide localized drug delivery therapy when triggered by electrophysiological conditions suggestive of an oncoming seizure. Robust investigational multifunctional neural probes are needed for the research required to realize these types of therapeutic devices.

One method to provide localized drug delivery to the CNS is through CED. ${ }^{2,20}$ The CED technique is the process of controlled infusion directly into brain parenchyma that is driven by a pressure source or pump. The volume of distribution into the tissue depends only on the injection volume. Furthermore, the concentration profile of the infusate is homogeneous within the volume of distribution and independent of infusion rate, duration, and volume. ${ }^{13}$ Over time, the concentration gradient between the volume of distribution and the remaining brain pa- renchyma further disperses the infusate by diffusion. Yet, the high degree of control afforded by CED methods is typically preferable to primarily diffusion-driven drug delivery, to match the high spatial resolution of microelectrode technology to the fluidic performance.

Several attempts have been made to integrate microfluidic drug delivery channels into microelectrode arrays. 5,19,30,32,35,36 These devices have the same microelectrode features used in BMI research, with the added ability to provide CED-driven drug infusion. However, much of this work has focused on the technical aspects of the fabrication process rather than the physiological performance of the device, due to the extremely stringent technical requirements needed to develop these technologies. Consequently, a simplified neural drug delivery probe ready for in vivo experimentation is needed for the data-driven development of more advanced microfabricated devices.

The objective of this work was to develop and to validate a neural probe design capable of simultaneous drug delivery and electrophysiological recordings in vivo. The device was designed to simplify CED of drugs for acute microelectrode array experiments. Proof-of-concept animal experiments were performed to assess drug dispersion and tissue damage, to determine the volume effects of infusion on neural activity, and to modulate neural activity through drug infusion. The results of these ex- 


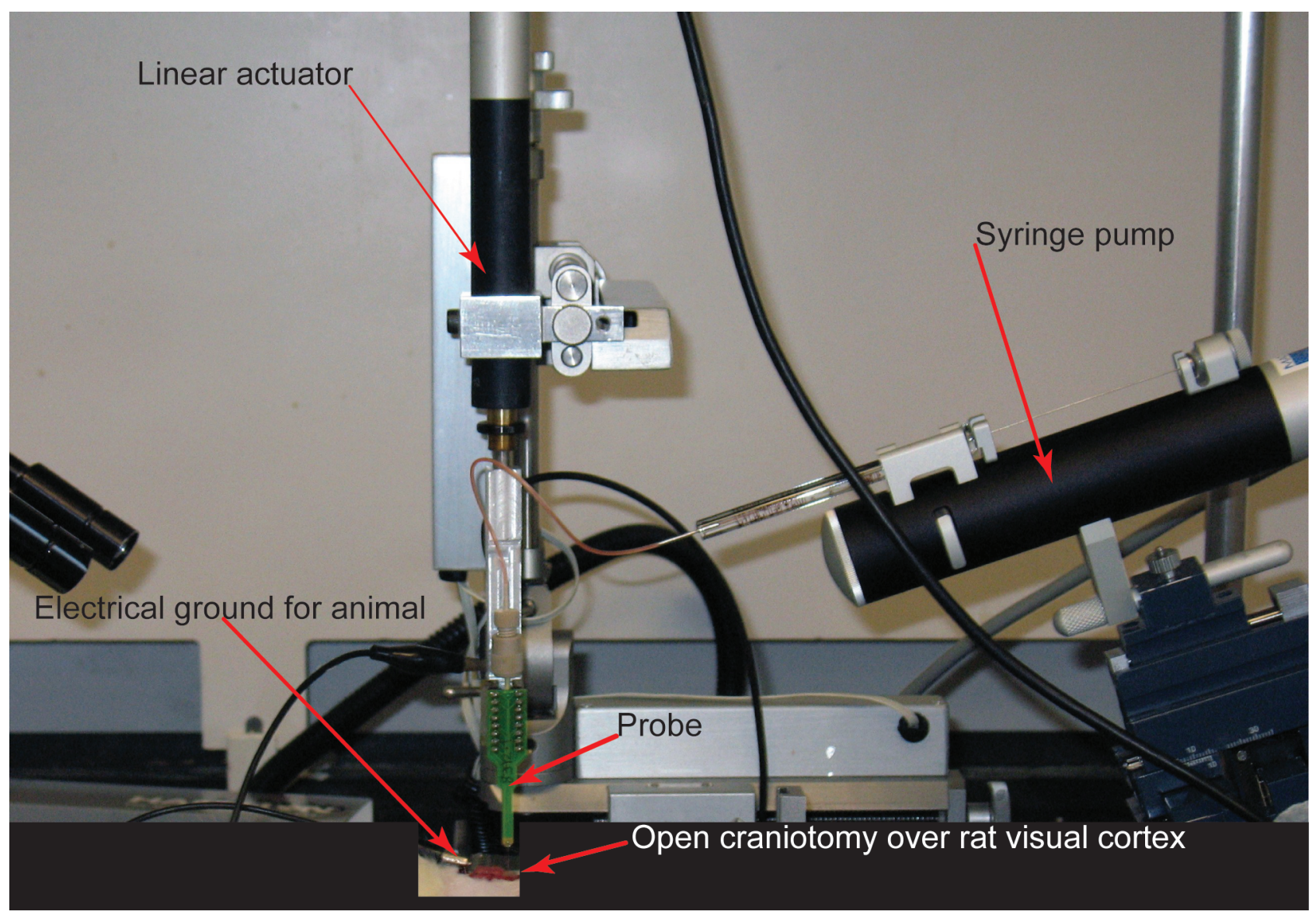

FIG. 2. Photograph showing the animal experimental setup with the neural probe inserted into rat cortex. The animal is secured in a stereotactic frame. The faraday cage and electronic amplifiers required for electrophysiological recordings are not shown.

periments allow us to determine if measured changes in neural activity are due to the direct actions of the infused drug or to nonspecific secondary effects. These proofof-concept experiments also demonstrate the increased functionality of concurrent electrophysiological and focal drug delivery, which not only monitor neural activity but also interface with the chemical environment, leading to greater understanding of how BMI devices function and expanding the potential BMI design space for clinical applications.

\section{Methods}

\section{Description of Multifunctional Neural Probe}

Multifunctional neural probes were created by incorporating a drug delivery catheter into a "chronic-style" Michigan microelectrode array on an acute experimental package (Fig. 1). Microelectrode arrays were fabricated using standard silicon processing techniques common for Michigan neural probes. ${ }^{18}$ The number of microelectrode sites and their spacing were selected to sample uniformly across the depth of rat cortex, and these dimensions are widely used in Michigan neural probe technology. This microelectrode design incorporated a flexible cable upstream of the implanted portion of the microelectrode ar- ray, which allowed the array to bend and mate to the drugdelivery catheter without breaking. ${ }^{16}$ The drug-delivery catheter and pump-connection adapters were bonded to the back of the device. This design was manufactured by NeuroNexus Technologies.

\section{Supporting Equipment}

Fluids were delivered through the catheter by using a $10-\mu l$ syringe (1700 Series, Hamilton Co.) driven by a microsyringe pump (UltraMicroPump 4, World Precision Instruments). For a $1-\mu l$ injection, the percent error in delivery volume was $0.133 \%(999 \pm 21 \mathrm{nl}$; mean $\pm \mathrm{SD}$ of 10 measurements). A programmable linear actuator was used (M-230.25, Physik Instrumente) to insert the probe into the brain. Automated implantation was preferred over manual insertion to minimize tissue damage. ${ }^{1}$

\section{Animal Preparation and Setup}

A total of 20 Sprague-Dawley rats (each weighing between 300 and $600 \mathrm{~g}$ ) was used for these experiments, following an approved protocol (\#08227) in accordance with the University Committee on Use and Care of Animals guidelines at the University of Michigan. The animals were housed and fed by university husbandry staff. All animal experiments involved nonsurvival surgery. 

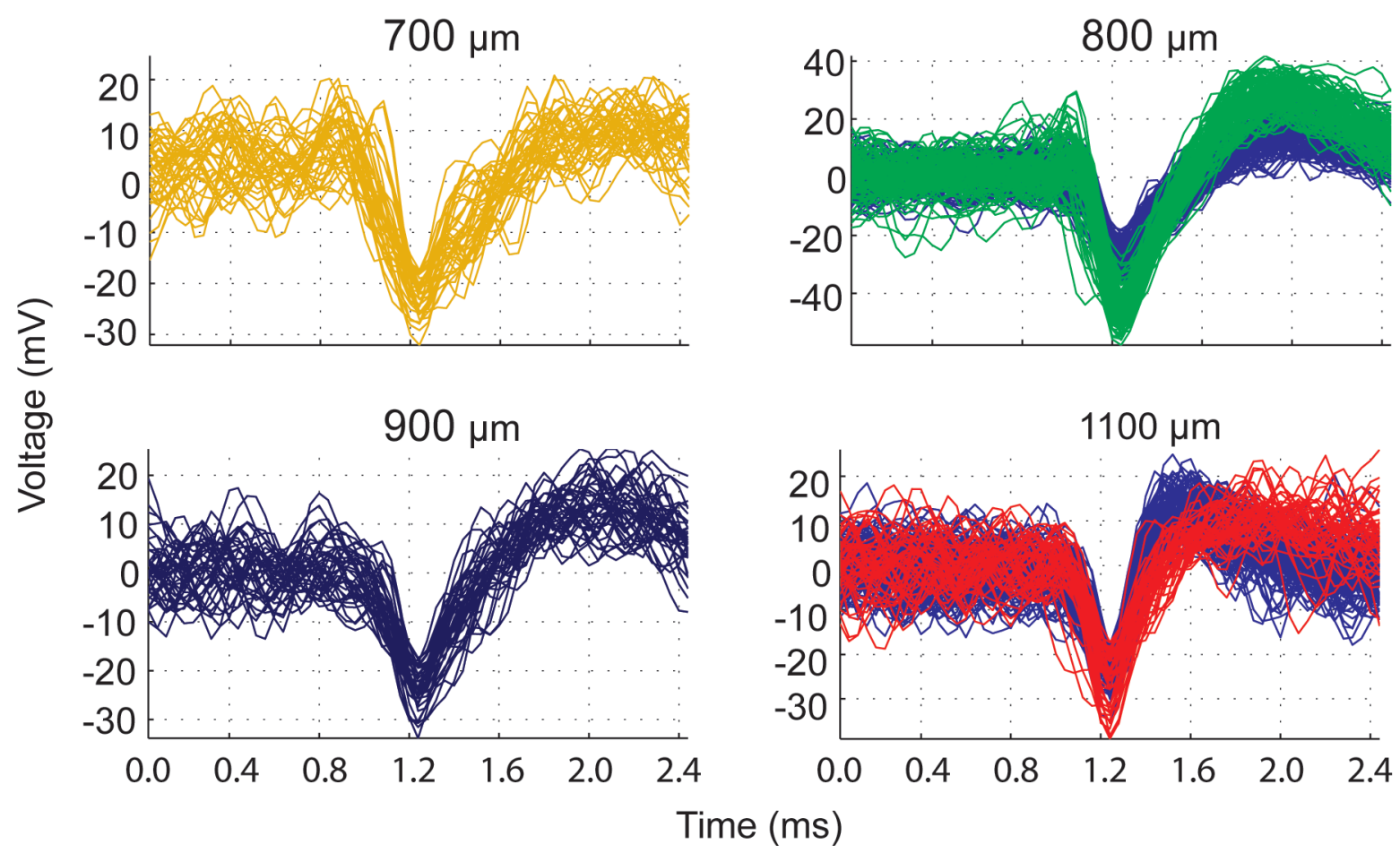

FIG. 3. An example of neural spikes recorded simultaneously on 4 of the 16 microelectrode sites of the device. Depth from the surface of the cortex is indicated for each channel. Two channels show 2 distinct patterns of unit activity as determined by the spike-sorting algorithm described in Methods.

Urethane $(1.25 \mathrm{~g} / \mathrm{kg}$ body weight) was used to provide prolonged anesthesia to the animal for the duration of the experiment. Urethane is commonly used because it minimally affects neurotransmitter levels involved in network oscillations in the brain. ${ }^{14,24,25}$ After the animal was secured in the stereotactic frame, craniotomies were opened bilaterally over primary visual cortex, located 6.5 $\mathrm{mm}$ posterior to bregma and $3 \mathrm{~mm}$ lateral to midline. The dura mater was resected to prevent the probe from buckling or breaking during insertion. After the surgery, the animal was placed in a faraday cage to minimize electrical noise, which often obscures spike activity. The linear actuator, microsyringe pump, and electrophysiological recording amplifiers were then added to the setup (Fig. 2). Supporting instrumentation was powered down to minimize noise during each recording session. A flashing light-emitting diode array with a 1-second on and 5 -seconds off cycle was placed in front of the animal to increase the probability of measuring driven action potentials in the visual cortex.

\section{Experimental Procedure}

The device was prefilled with the infusate before insertion so that air would not be injected into the brain. The solution was not warmed to body temperature because it would return to room temperature prior to infusion due to the small volume used $(<100 \mu \mathrm{l})$ and the required setup time (20-30 minutes). The probe was positioned over the craniotomy, touching the surface of the brain, by using the stereotactic manipulator, and it was inserted with the linear actuator to an initial depth of $2 \mathrm{~mm}$ at a rate of
$1.2 \mathrm{~mm} / \mathrm{second}$. If no action potentials were identified at that depth, the probe was either advanced or withdrawn until spiking activity was measured. The catheter outlet placement was limited to a range $1.5-5 \mathrm{~mm}$ below the surface of the cortex. Only a single $1-\mu \mathrm{l}$ infusion at a rate of $100 \mathrm{nl} /$ minute was delivered into each craniotomy for any given trial. These parameters were selected based on prior benchtop work, which is briefly summarized in the Results section. Electrophysiological data were typically recorded before, during, and after drug infusion.

\section{Electrophysiological Recordings}

For all animals in this study, electrophysiological data were acquired using a TDT Pentusa Recording System (Tucker-Davis Technologies). These neuronal signals were acquired through the 16-channel electrodes with a head-stage buffer amplifier to avoid signal loss in data transmission. Signals were sequentially filtered by an antialiasing filter in the preamplifier, digitized at a $\sim 25$ $\mathrm{kHz}$ sampling rate, and digitally band-pass filtered from 2 to $5000 \mathrm{~Hz}$. Wideband signals were acquired to capture both spiking and LFP activity. Signals were continuously recorded in segments ranging from 30 seconds to $>10$ minutes in duration. To reduce noise in the recordings further, common average referencing was used in real time..$^{22}$ This technique calculates a reference signal by averaging data on every channel, and subtracts this value from each electrode recording to eliminate noise that is correlated across the array. Common average referencing is necessary for real-time drug delivery recordings, because the active syringe pump is a large source of elec- 


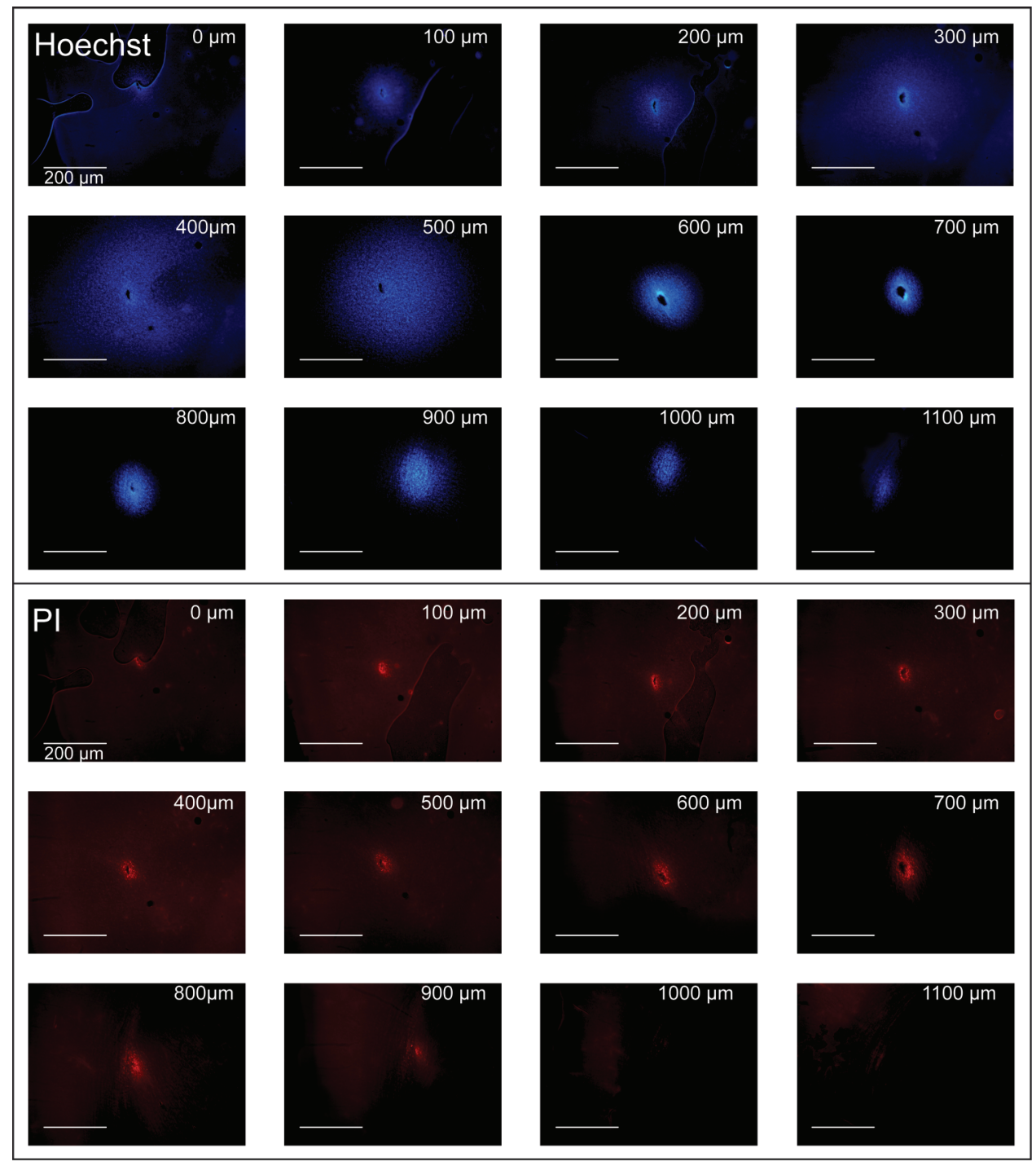

FIG. 4. Photomicrographs showing the histological findings in a single injection site of Hoechst (upper panels) and PI (lower panels) viewed using an epifluorescence wide-field microscope at $\times 50$ magnification. The brain tissue was sectioned in transverse slices relative to the long axis of the neural device. Depth is labeled with respect to the first tissue slice with visible dye. The same tissue slice is shown for both stains for a given depth. The device outlet location cannot be localized to a specific tissue slice. Some images show artifact due to the slide fixation process.

trical noise. Neuronal activity was better assessed during experimentation by performing this technique in real time, and helped guide probe insertion depth.

\section{In Vivo Experiments}

Three different animal experiments were conducted to evaluate device function in vivo. The purpose of the first experiment was to assess drug dispersion and tissue damage by imaging infused stains. The infusate consisted of a solution containing Hoechst 33342 and PI (both from Invitrogen Corp.), each at $1 \mathrm{mg} / \mathrm{ml}$ in aCSF (Harvard Apparatus). The purpose of the second experiment was to determine if the volume effects of the infusate would alter measured neural activity. For this experiment, $1 \mu \mathrm{l}$ of aCSF was delivered through the device into brain tissue. The purpose of the third experiment was to observe changes in neuronal activity due to a delivered drug. For this experiment, TTX (Tocris Bioscience) at $10 \mathrm{ng} / \mu \mathrm{l}$ was added to the solution used in the first experiment and delivered through the device into brain tissue.

\section{Histological Studies}

After each experiment that required tissue assessment, the animal was perfused with $4 \%$ paraformaldehyde for histological studies. After perfusion, the brain was excised from the skull and refrigerated at $4^{\circ} \mathrm{C}$ in $4 \%$ paraformaldehyde for at least 24 hours. A vibratome (myNeuroLab) was used to make $100-\mu \mathrm{m}$-thick transverse brain slices that were mounted to slides by using Prolong Gold Antifade Reagent (Invitrogen Corp.). After allowing the slides to fix for at least 24 hours, the tissue slices were viewed using an epifluorescence wide-field microscope (MZFLIII, Leica). A 12-bit color charge-coupled device camera (CoolSNAP PRO Color, MediaCybernet- 
A) aCSF delivery

$1400 \mu \mathrm{m}$

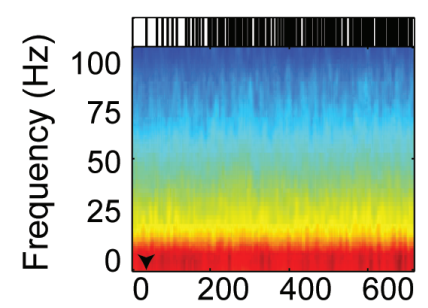

$1300 \mu \mathrm{m}$

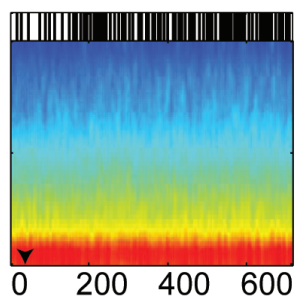

$800 \mu \mathrm{m}$

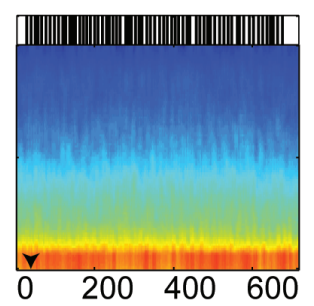

Time (sec)
B) TTX delivery

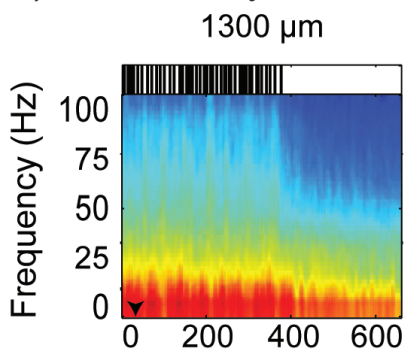

$1200 \mu \mathrm{m}$

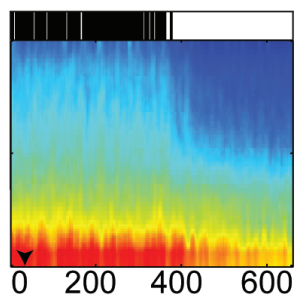

$1100 \mu \mathrm{m}$

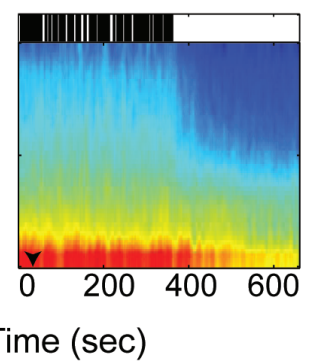

$900 \mu \mathrm{m}$

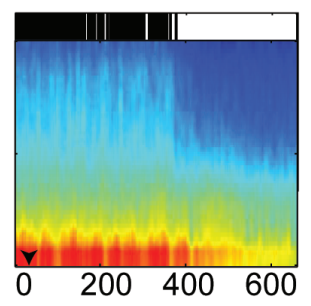

$0 \quad 200 \quad 400 \quad 600$
$400 \mu \mathrm{m}$

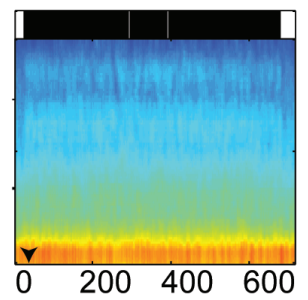

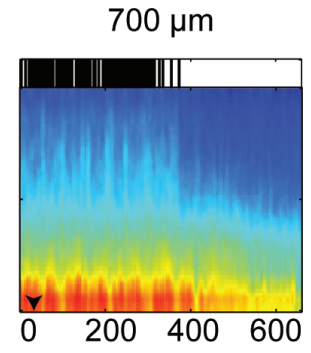

FIG. 5. The LFP spectrograms and raster plots obtained during the delivery of aCSF (A) and TTX (B) for sites with identified units. The start of drug delivery is marked by a triangle on the $\mathrm{x}$ axis, which begins $\sim 30$ seconds into the recording block and continues for 600 seconds. Each site is labeled in relation to its distance from the catheter outlet. Each raster plot shows every 10th identified spike. Power is presented on the same scale for all sites of a given trial; hot (red) colors indicate higher power and cold (blue) colors indicate lower power. The spectrograms for microelectrode sites without identified units were similar to channels with identified units for both aCSF and TTX delivery (not shown). Note the deactivation of neural activity during TTX delivery at $\sim 400$ seconds.

ics) mounted to the microscope was used to store each image digitally. Only tissue slices with dye visible to the eye through the microscope were imaged. The exposure time for each image was adjusted such that the maximum signal intensity matched the saturation point of the charge-coupled device camera. The image background was normalized in postprocessing software (Photoshop, Adobe Systems).

\section{Electrophysiological Analysis}

Neural recording data were analyzed after acquisition by using custom automated MATLAB (Mathworks, Inc.) software, as described in detail elsewhere. ${ }^{22,23} \mathrm{As}$ an overview, the wide-band recordings were filtered in software to isolate the spike data $(300-5000 \mathrm{~Hz})$ from the LFP data $(1-100 \mathrm{~Hz})$. To identify individual units, the threshold for the high-frequency data was established by using a window set at 3.5 SDs below the mean of the data. A 2.4-msec waveform was extracted from the data stream at each threshold crossing. To group isolated waveforms to a single neuronal unit, principal component analysis was then completed, and the resultant components were separated into individual clusters by using Fuzzy C-means clustering. The results of this spike-sorting process from a data set within this study are shown in Fig. 3.

The LFPs were primarily analyzed in the frequency domain. Power spectrums and spectrograms were calculated using the signal processing toolbox within MAT-
LAB. For a comparative analysis, data for all channels of a given trial were combined and the LFP power was averaged to create delta ( 0 to $<3 \mathrm{~Hz}$ ), theta ( 3 to $<7 \mathrm{~Hz}$ ), alpha $(7$ to $<12 \mathrm{~Hz})$, beta $(12$ to $<30 \mathrm{~Hz})$, and gamma bands $(30-100 \mathrm{~Hz})$. The mean value for each band before and 1 hour after infusion was compared using a paired t-test.

\section{Results}

This study was performed using the multimodal neural probe illustrated in Fig. 1 and with the setup shown in Fig. 2, as described in the Methods. Figure 3 demonstrates the quality of units recorded with the device and the effectiveness of the spike-sorting algorithms.

\section{Validation and Optimization of Drug Delivery Parameters}

Prior work was performed to validate the probe design and performance in $0.6 \%$ agarose gel to determine appropriate probe insertion and drug infusion rates. This concentration of agarose has poroelastic properties that are similar to brain parenchyma with respect to volume of delivery, infusion pressure, and insertion force. ${ }^{8}$ Results (not shown) from the agarose tests showed that slower infusion rates decreased backflow, permitting the catheter outlet to act more like a point source. Additionally, faster probe insertion speeds minimized agarose damage by cutting sharply into the gel rather than creating large deformations and tears. Damage disrupts the tissue-device 


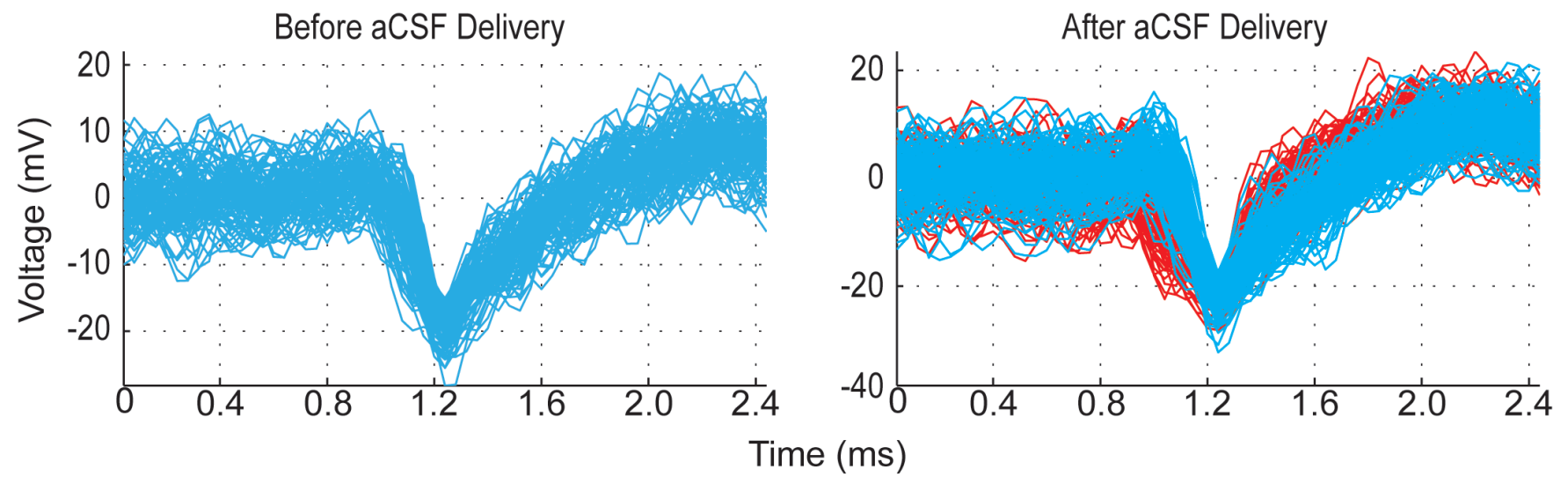

FIG. 6. Tracings showing the morphological features of a unit before and after aCSF delivery. In this example, a second unit was identified after aCSF delivery.

interface, creating a low-resistance fluid shunt along the insertion path. This allows the infusate to reflux outside of the cortex instead of penetrating into the brain parenchyma. These results are consistent with findings from other studies on the optimization of CED parameters in cortex. ${ }^{6,27}$ These studies demonstrate that backflow is significantly increased for infusion rates $>1 \mu \mathrm{l} /$ minute versus infusion rates $<0.5 \mu \mathrm{l} /$ minute. They also show that larger cannula sizes increase backflow, but tissue relaxation time after device insertion did not affect backflow. Consequently, all animal experiments for this study were performed using an infusion rate of $100 \mathrm{nl} /$ minute and an insertion speed of $1.2 \mathrm{~mm} / \mathrm{second}$, which was the fastest speed of the linear actuator.

\section{Assessment of Drug Dispersion and Tissue Damage}

Hoechst and PI stains were successfully visualized in brain tissue after histological studies were performed (Fig. 4). Hoechst and PI both intercalate with DNA, but PI is membrane impermeable, and thus only stains cells that have damaged cellular membranes. ${ }^{36}$ Consequently, tissue stained with Hoechst reflects drug dispersion within the brain, whereas PI only highlights areas of tissue damage. Hoechst was used as a visible proxy for TTX distribution in tissue, because both are hydrophilic and have molecular weights on the same order of magnitude. Hoechst dispersed in a roughly spherical distribution through the tissue elongated along the axis of the probe, indicating that the infusate penetrated into brain parenchyma rather than primarily shunting outside the brain along the insertion track. Furthermore, the dye did not stain slices extending to the surface of the brain. The area of tissue stained with PI was tightly focused around the probe insertion track, and was markedly reduced from the area stained with Hoechst. Therefore, the volume of tissue damaged by the device was less than the volume of drug distribution.

\section{Effects of aCSF and TTX During Infusion}

Real-time electrophysiological data during aCSF and TTX delivery were successfully recorded, demonstrating the ability of the device to record electrophysiological data and deliver drugs simultaneously. Infusion started 30 seconds into the recording window and continued for
600 seconds. Representative data from single aCSF and TTX infusions are shown in Fig. 5 for electrode sites with identified units. Only a single unit is illustrated for channels with multiple units. Each electrode site is represented by a spectrogram and raster plot. The spectrograms show the LFP power as a function of time and frequency. A raster plot is placed above each spectrogram and displays a single-unit firing event as a vertical line. The line density reflects the firing rate of each neuron.

The drug TTX strongly inhibits voltage-gated sodium channels, thus stopping action potential propagation along all neuronal axons. ${ }^{28,29}$ Neuroscientists use TTX to create reversible lesions to better study nervous system function. ${ }^{3,12,26,34,40,42}$ Tetrodotoxin was selected as the delivery drug because it changes neuronal activity in a manner that is straightforward to interpret. Neither the LFP power nor the spiking activity changed during the aCSF infusion, but both measures dramatically changed during TTX delivery. All spiking activity stopped $\sim 400$ seconds into the TTX infusion, which corresponds to a delivered volume near $0.66 \mu \mathrm{l}$. The LFP power dropped at this time point and continued to do so throughout the remainder of the recording window. Because aCSF did not alter unit activity, changes in activity can be attributed to drug delivery.

\section{Spike Data Before and After Infusion}

Spike data were compared before aCSF and TTX delivery and 1 hour after delivery. Spikes were identified before and after aCSF delivery. Units showed a similar waveform shape at a given microelectrode site, suggesting that the same neuron was active during both recording segments (Fig. 6). No spikes were identified after TTX delivery. This is consistent with results showing that spiking activity stopped during TTX infusion. The addition of TTX reversibly inhibits neuronal activity; however, the recovery time course for a $1-\mu \mathrm{l}$ injection at $10 \mathrm{ng} / \mu \mathrm{l}$ can be as long as 20-24 hours. ${ }^{42}$ Consequently, the return of neuronal activity was neither observed nor expected 1 hour after TTX delivery.

\section{The LFP Data Before and After Infusion}

The LFP data were also compared before and 1 hour 
A) LFP power before and after aCSF infusion

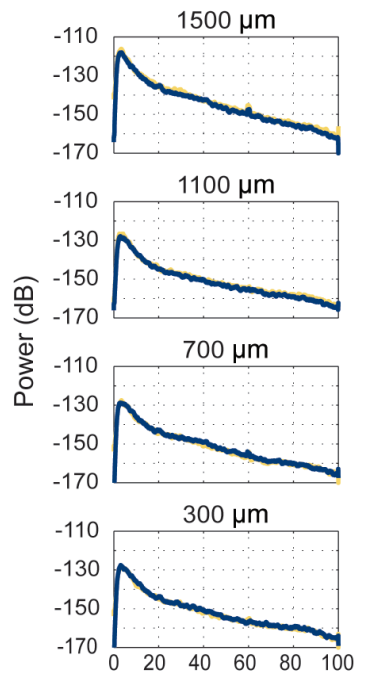

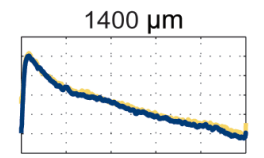

$1000 \mu \mathrm{m}$

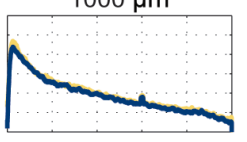

$600 \mu \mathrm{m}$

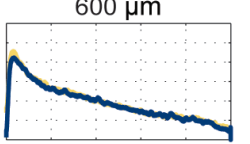

$200 \mu \mathrm{m}$

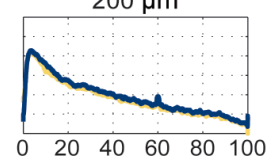

$1300 \mu \mathrm{m}$

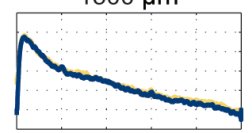

$900 \mu \mathrm{m}$

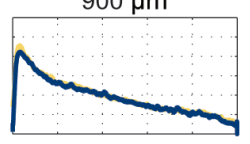

$500 \mu \mathrm{m}$

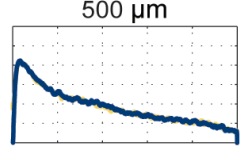

$100 \mu \mathrm{m}$

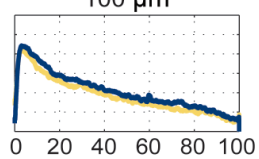

$(\mathrm{Hz})$

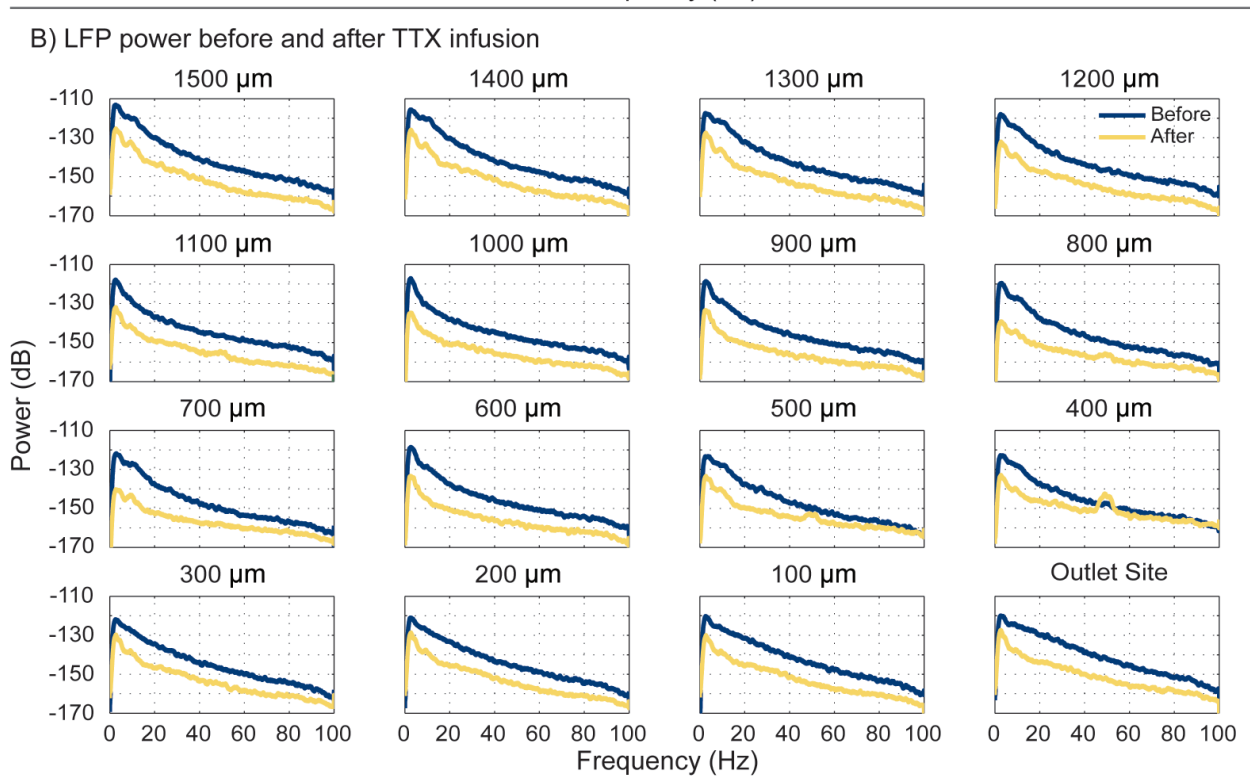

FIG. 7. Recordings showing changes in LFP power before and 1 hour after aCSF (A) and TTX (B) infusion. Each site is labeled in relation to its distance from the catheter outlet. A $60-\mathrm{Hz}$ electrical noise is evident on several electrode sites. Note that TTX delivery caused an appreciable decrease in power at every site, whereas aCSF delivery caused no notable change in LFP power.

after aCSF and TTX delivery. Data for 2 different trials of aCSF and TTX infusions were aggregated for a statistical comparison of a change in LFP power before and after infusion by using a paired t-test (Table 1). The aCSF infusion did not create a statistically significant difference in power for any frequency band. Conversely, TTX infusion created a statistically significant $(\mathrm{p}<0.05)$ change in power in every frequency band. Changes in LFP were not obviously different at microelectrode sites closer to the catheter opening (Fig. 7).

\section{Discussion}

The multifunctional neural probe used in this study successfully modified neuronal activity through drug delivery as measured by electrophysiological monitoring. However, the drug-delivery resolution for the parameters studied is less than electrophysiological resolution of the device. This is reflected by the uniform change in electrophysiological data measured in response to drug delivery on every site of the microelectrode array. More tightly controlled drug delivery is needed to compare electrophysiological recordings from sites measuring neural activity modulated by the drug to other sites on the array measuring unaltered neural activity during the same experiment. Alternatively, a device with fewer or more widely spaced microelectrode sites can be used to avoid taking recordings that do not provide additional information about drug kinetics, delivery, or neuronal modulation. 
TABLE 1: Comparison of LFP power before and $~ 1$ hour after aCSF and TTX delivery

\begin{tabular}{lll}
\hline & \multicolumn{2}{c}{$p$ Value* } \\
\cline { 2 - 3 } Frequency Band $(\mathrm{Hz})$ & aCSF & TTX \\
\hline delta $(0$ to $<3)$ & 0.419 & $<0.05$ \\
theta $(3$ to $<7)$ & 0.763 & $<0.05$ \\
alpha $(7$ to $<12)$ & 0.580 & $<0.05$ \\
beta $(12$ to $<30)$ & 0.448 & $<0.05$ \\
gamma $(30-100)$ & 0.298 & $<0.05$ \\
\hline
\end{tabular}

* The $p$ values were calculated using a paired t-test. Data were pooled from 2 different injections with a 16-channel microelectrode array to provide a sample size of 32 microelectrodes for each statistical comparison.

\section{Assessment of Drug Dispersion and Tissue Damage}

The method to visualize drug delivery demonstrates that the infusate penetrated into the brain tissue with negligible backflow. However, the elliptical drug distribution pattern suggests that the catheter outlet could not be idealized as a point source within this study. For better planning and completion of future experiments, a model is needed to correlate the injection volume with the volume of distribution in tissue. Biotransport in the brain is complicated by various processes, including diffusion, bulk flow, molecular uptake, metabolism, and the device-tissue interface. ${ }^{31,39}$ Several efforts have been made to describe drug distribution for CED mathematically, and can be applied to drug delivery with this device as well.,21,27

Tissue damage was evident with the addition of PI. It is not understood if the tissue damage around the probe is dependent on the cross-sectional area and if larger catheter sizes decrease the ability to measure units. The multifunctional electrode had a cross-sectional area of $>20,000$ $\mu \mathrm{m}^{2}$, which is dramatically larger than the typical crosssectional area for a Michigan microelectrode array of $1500 \mu \mathrm{m} .{ }^{2,15}$ Although no quantitative statistical analyses were performed for comparison, electrophysiological performance for the multifunctional device was comparable to the Michigan microelectrode array, despite the larger size, based on experience within our laboratory. A better understanding of tissue damage would help steer device design and help determine if the smaller and technically more involved microfabricated fluidic channels are necessary to improve multifunctional device performance.

\section{Volume Effects of Infusion on Neural Activity}

Because neuronal activity was not changed with aCSF delivery, potential tissue damage due to fluid delivery may not be physiologically significant as assessed by electrophysiological testing for the parameters studied (1 $\mu \mathrm{l}$ at $100 \mathrm{nl} / \mathrm{minute})$. In fact, the upper limit of pressure injections into cortex before tissue damage is thought to range from 12 to $20 \mu 1 .{ }^{9}$ The trauma of the device insertion did not completely damage the surrounding neuronal tissue, as evident by the ability to record spikes prior to drug delivery. It is not clear whether tissue damage was due solely to probe insertion or if it was partially depen- dent on fluid delivery. Possible neuronal displacement or changes in intracranial pressure may not be physiologically relevant. Because the dura mater is open, the infusate can shunt or the brain can herniate outside of the craniotomy to dissipate pressure and allow tissue around the probe to relax. This area requires more investigation because implanted drug delivery devices will require the craniotomy to be sealed from the outside environment.

\section{Drug-Induced Modulation of Neural Activity}

The infusion of TTX successfully suppressed neuronal activity in the vicinity of the neural probe. The TTX suppressed all spiking activity during infusion and lowered LFP power. These changes started roughly twothirds of the way through the infusion at every electrode depth, indicating that tissue around each electrode site was exposed to TTX in the same time frame. The tissue at the catheter outlet could be acting like a valve that does not open until sufficient backpressure is created. Backflow may also act first to shunt TTX along the probe track and then to suppress neuronal activity immediately next to the probe at a faster time course than drug penetration into brain parenchyma, due to a difference in flow resistivity. Additionally, because spikes are typically recorded from a distance of up to $50 \mu \mathrm{m}$ from the electrode array, this signal would first be suppressed by the backflow of TTX. The LFPs are recorded from a radius of several hundred microns away from the electrode array, and thus changes in this signal may be more indicative of infusate penetration into brain tissue and drug kinetics. If the catheter outlet acted as an ideal point source with appropriately selected drug delivery parameters, changes in neuronal activity should first be measured at electrode sites most proximal to the outlet and propagate to distal sites due to the high spatial resolution of the array. The delivery rate for these experiments was selected to minimize backflow of infusate to the surface of the cortex, but an even tighter control of backflow is needed to see a progressive change of neuronal modulation across the electrode sites. Consequently, an even slower infusion rate should be used to minimize backflow and backpressure effects further, by allowing the infusate to penetrate into tissue, thus allowing the outlet to behave more closely to an ideal point source.

Measured action potentials were only suppressed during infusion, thus indicating that drug diffusion from the outlet did not substantially change the activity of neurons in the vicinity of the microelectrode array. Yet, diffusion may limit the use of this device in studies that require very tight control of drug delivery. Devices that rely on diffusion-based drug delivery create a volume of distribution extending $400 \mu \mathrm{m}$ radially, which is larger than the typical microelectrode site spacing. ${ }^{37}$ Microfabricated devices have been created with valves that minimize the potential effects of diffusion, but their effectiveness has not been evaluated in vivo with respect to electrophysiological measures. ${ }^{32}$

\section{Conclusions}

This device provides a platform to drive further de- 


\section{P. Rohatgi et al.}

velopments in drug delivery and in the manufacture of clinically useful multifunctional neural prostheses. As BMI technology matures, drug delivery can provide an additional means to interface with the CNS, either to improve system performance or to provide therapy. Additionally, the ability of the device to monitor electrophysiological changes in real time in response to drug delivery will become a powerful investigational tool in neuroscience and pharmacology.

Studies to investigate different device designs, including varied catheter size, outlet geometries, and delivery parameters, should be used for further advancement in microfabricated multifunctional probe design. Additionally, polymer-based microelectrode array technology should soon replace the use of silicon substrate electrodes. This change will improve device usability by allowing the catheter to bend without breaking the bonded microelectrode array. Efforts are also now actively underway to modify the device to create a fully implantable long-term drug delivery system.

\section{Disclosure}

Dr. Daryl R. Kipke has a significant financial interest in NeuroNexus Technologies. Nicholas B. Langhals is a part-time consultant at NeuroNexus Technologies. Dr. Patil and Mr. Rohatgi have no conflicts to disclose.

The authors acknowledge the financial support (Grant No. P41 EB002030) of the Center for Neural Communication Technology (a P41 Resource Center funded by the National Institute of Biomedical Imaging and Bioengineering [NIBIB], and supported by the National Institutes of Health). They also thank the Student Biomedical Research Program (which provided specific support to Mr. Rohatgi), funded through the University of Michigan Medical School.

\section{Acknowledgments}

The authors thank Dr. Kip Ludwig for project planning and manuscript editing assistance, and Denzel Davis, Matthew Gibson, and the other members of the Neural Engineering Laboratory of the University of Michigan for their assistance with this study.

\section{References}

1. Bjornsson CS, Oh S, Al-Kofahi Y, Lim Y, Smith K, Turner J, et al: Effects of insertion conditions on tissue strain and vascular damage during neuroprosthetic device insertion. J Neural Eng 3:196-207, 2006

2. Bobo RH, Laske DW, Akbasak A, Morrison PF, Dedrick RL, Oldfield EH: Convection-enhanced delivery of macromolecules in the brain. Proc Natl Acad Sci U S A 91:2076-2080, 1994

3. Boehnke SE, Rasmusson DD: Time course and effective spread of lidocaine and tetrodotoxin delivered via microdialysis: an electrophysiological study in cerebral cortex. J Neurosci Methods 105:133-141, 2001

4. Chapin JK, Moxon KA, Markowitz RS, Nicolelis MAL: Realtime control of a robot arm using simultaneously recorded neurons in the motor cortex. Nat Neurosci 2:664-670, 1999

5. Chen J, Wise KD, Hetke JF, Bledsoe SC Jr: A multichannel neural probe for selective chemical delivery at the cellular level. IEEE Trans Biomed Eng 44:760-769, 1997

6. Chen MY, Lonser RR, Morrison PF, Governale LS, Oldfield EH: Variables affecting convection-enhanced delivery to the striatum: a systematic examination of rate of infusion, cannula size, infusate concentration, and tissue-cannula sealing time. J Neurosurg 90:315-320, 1999
7. Chen ZJ, Broaddus WC, Viswanathan RR, Raghavan R, Gillies GT: Intraparenchymal drug delivery via positive-pressure infusion: experimental and modeling studies of poroelasticity in brain phantom gels. IEEE Trans Biomed Eng 49:85-96, 2002

8. Chen ZJ, Gillies GT, Broaddus WC, Prabhu SS, Fillmore H, Mitchell RM, et al: A realistic brain tissue phantom for intraparenchymal infusion studies. J Neurosurg 101:314-322, 2004

9. Demer JL, Robinson D: Effects of reversible lesions and stimulation of olivocerebellar system on vestibuloocular reflex plasticity. J Neurophysiol 47:1084-1107, 1982

10. Donoghue JP: Connecting cortex to machines: recent advances in brain interfaces. Nat Neurosci 5 (Suppl): 1085-1088, 2002

11. Fitzsimmons NA, Drake W, Hanson TL, Lebedev MA, Nicolelis MA: Primate reaching cued by multichannel spatiotemporal cortical microstimulation. J Neurosci 27:5593-5602, 2007

12. Gallo M, Bielavska E, Roldán G, Bures J: Tetrodotoxin inactivation of the gustatory cortex disrupts the effect of the Nmethyl-aspartate antagonist ketamine on latent inhibition of conditioned taste aversion in rats. Neurosci Lett 240:61-64, 1998

13. Groothuis DR: The blood-brain and blood-tumor barriers: a review of strategies for increasing drug delivery. Neuro Oncol 2:45-59, 2000

14. Hara K, Harris RA: The anesthetic mechanism of urethane: the effects on neurotransmitter-gated ion channels. Anesth Analg 94:313-318, 2002

15. Hetke J, Anderson D, Wise K: Design ranges for silicon multichannel neural probes, in Engineering in Medicine and Biology Society, 1996. Bridging Disciplines for Biomedicine. Proceedings of the 18th Annual International Conference of the IEEE Engineering in Medicine and Biology Society, Amsterdam, 1996, Vol 1. Los Alamitos, CA: IEEE Publications, pp 266-267

16. Hetke JF, Lund J, Najafi K, Wise K, Anderson D: Silicon ribbon cables for chronically implantable microelectrode arrays. IEEE Trans Biomed Eng 41:314-321, 1994

17. Kipke DR, Shain W, Buzsaki G, Fetz E, Henderson JM, Hetke JF, et al: Advanced neurotechnologies for chronic neural interfaces: new horizons and clinical opportunities. J Neurosci 28:11830-11838, 2008

18. Kipke DR, Vetter RJ, Williams JC, Hetke JF: Silicon-substrate intracortical microelectrode arrays for long-term recording of neuronal spike activity in cerebral cortex. IEEE Trans Neural Syst Rehabil Eng 11:151-155, 2003

19. Li Y, Baek K, Gulari M, Wise K: A drug-delivery probe with an in-line flowmeter based on trench refill and chemical mechanical polishing techniques. Sensors, 2007 IEEE: 11441147,2007

20. Lieberman DM, Laske DW, Morrison PF, Bankiewicz KS, Oldfield EH: Convection-enhanced distribution of large molecules in gray matter during interstitial drug infusion. J Neurosurg 82:1021-1029, 1995

21. Linninger AA, Somayaji MR, Mekarski M, Zhang L: Prediction of convection-enhanced drug delivery to the human brain. J Theor Biol 250:125-138, 2008

22. Ludwig KA, Miriani RM, Langhals NB, Joseph MD, Anderson DJ, Kipke DR: Using a common average reference to improve cortical neuron recordings from microelectrode arrays. J Neurophysiol 101:1679-1689, 2009

23. Ludwig KA, Uram JD, Yang J, Martin DC, Kipke DR: Chronic neural recordings using silicon microelectrode arrays electrochemically deposited with a poly(3,4-ethylenedioxythiophene) (PEDOT) film. J Neural Eng 3:59-70, 2006

24. Maggi C, Meli A: Suitability of urethane anesthesia for physiopharmacological investigations in various systems. Part 1: general considerations. Experientia 42:109-114, 1986 


\section{Microelectrode neural probe with integrated drug delivery}

25. Maggi C, Meli A: Suitability of urethane anesthesia for physiopharmacological investigations in various systems. Part 2: cardiovascular system. Experientia 42:292-297, 1986

26. Martin JH, Ghez C: Pharmacological inactivation in the analysis of the central control of movement. J Neurosci Methods 86:145-159, 1999

27. Morrison PF, Chen MY, Chadwick RS, Lonser RR, Oldfield EH: Focal delivery during direct infusion to brain: role of flow rate, catheter diameter, and tissue mechanics. Am J Physiol 277:R1218-R1229, 1999

28. Narahashi T, Moore JW, Scott WR: Tetrodotoxin blockage of sodium conductance increase in lobster giant axons. J Gen Physiol 47:965-974, 1964

29. Narahashi T: Mechanism of action of tetrodotoxin and saxitoxin on excitable membranes. Fed Proc 31:1124-1132, 1972

30. Neeves KB, Lo CT, Foley CP, Saltzman WM, Olbricht WL: Fabrication and characterization of microfluidic probes for convection enhanced drug delivery. J Control Release 111:252-262, 2006

31. Nicholson C: Diffusion and related transport mechanisms in brain tissue. Rep Prog Phys 64:815-884, 2001

32. Papageorgiou D, Shore S, Bledsoe S Jr, Wise K: A shuttered neural probe with on-chip flowmeters for chronic in vivo drug delivery. J Microelectromech Syst 15:1025-1033, 2006

33. Polikov VS, Tresco PA, Reichert WM: Response of brain tissue to chronically implanted neural electrodes. J Neurosci Methods 148:1-18, 2005

34. Popelar J, Nwabueze-Ogbo FC, Syka J: Changes in neuronal activity of the inferior colliculus in rat after temporal inactivation of the auditory cortex. Physiol Res 52:615-628, 2003

35. Rathnasingham R, Kipke DR, Bledsoe SC Jr, McLaren JD: Characterization of implantable microfabricated fluid delivery devices. IEEE Trans Biomed Eng 51:138-145, 2004

36. Retterer ST, Smith K, Bjornsson C, Turner J, Isaacson M, Shain W: Constant pressure fluid infusion into rat neocortex from implantable microfluidic devices. J Neural Eng 5:385391,2008
37. Retterer ST, Smith KL, Bjornsson CS, Neeves KB, Spence AJ, Turner JN, et al: Model neural prostheses with integrated microfluidics: a potential intervention strategy for controlling reactive cell and tissue responses. IEEE Trans Biomed Eng 51:2063-2073, 2004

38. Spataro L, Dilgen J, Retterer S, Spence AJ, Isaacson M, Turner JN, et al: Dexamethasone treatment reduces astroglia responses to inserted neuroprosthetic devices in rat neocortex. Exp Neurol 194:289-300, 2005

39. Sykova E, Nicholson C: Diffusion in brain extracellular space. Physiol Rev 88:1277-1340, 2008

40. van Duuren E, van der Plasse G, van der Blom R, Joosten RN, Mulder AB, Pennartz CM, et al: Pharmacological manipulation of neuronal ensemble activity by reverse microdialysis in freely moving rats: a comparative study of the effects of tetrodotoxin, lidocaine, and muscimol. J Pharmacol Exp Ther 323:61-69, 2007

41. Velliste M, Perel S, Spalding MC, Whitford AS, Schwartz AB: Cortical control of a prosthetic arm for self-feeding. Nature 453:1098-1101, 2008

42. Zhuravin IA, Bures J: Extent of the tetrodotoxin induced blockade examined by pupillary paralysis elicited by intracerebral injection of the drug. Exp Brain Res 83:687-690, 1991

Manuscript submitted March 15, 2009.

Accepted April 22, 2009.

Portions of this work were presented in poster form at the 2008 Fall Student Biomedical Research Forum and the 2008 Engineering Graduate Symposium, both held in Ann Arbor, Michigan, in November 2008.

Address correspondence to: Pratik Rohatgi, M.S.E., Room 2247, Ann \& Robert H. Lurie Biomedical Engineering Building, University of Michigan, 1101 Beal Avenue, Ann Arbor, Michigan 48109. email: prohatgi@umich.edu. 